\title{
A Taxonomy of Lexical Strategies of Chinese Learners of English ${ }^{*}$
}

\author{
CHEN Long \\ Jinan University, Guangzhou, China
}

\begin{abstract}
Lexical strategies of Chinese learners of English are frequently observed in learning and using the English language. In this paper, the author designed an experiment to collect empirical data in order to find whether the Chinese learners of English realize the use of lexical strategies when they encounter communication problems. The data were collected by employing think-aloud protocols and retrospective interviews in translation from Chinese into English. The translation drafts and clean copies were also utilized for identifying lexical strategies by Chinese learners of English. Through analyzing the data collected in the experiment, the strategies are identified and classified into four categories, namely Retrieval, Strategic activities, Non-strategic activities/Monitoring strategies, and Abandonment. In the taxonomy of lexical strategies, three of the categories consist of sub-categorization with retrieval as an exception. Although the strategies elicited in the study cannot cover all scopes of English language learning and communication, they can be considered as findings that offer the learners and teachers of English guidance to use in overcoming communicative difficulties. The strategies demonstrated in the taxonomy also show us that we need to realize some lexical strategies so that communication can be carried on fluently and meaningfully.
\end{abstract}

Keywords: taxonomy, lexical strategies, Chinese learners of English, think-aloud protocols, retrospective interviews, translation, communication

\section{Introduction}

The study of Chinese learners of English in translation from Chinese into English reveals that different lexical strategies are employed. By analyzing introspective and retrospective data of advanced and intermediate learners in the experiment, lexical strategies are identified but conceptually reorganized based on existing literature, most notably Zimmermann (1989b) and Poulisse, Bongaerts and Kellerman (1990, chapter 6). The identification of lexical strategies is described by referring to think-aloud protocols, retrospective interviews, drafts, and clean copies collected as data in the study which aims at investigating lexical strategies employed by Chinese learners of English when they have difficulties in communication. Examples are provided for describing

\footnotetext{
* Acknowledgements: The findings of lexical strategies would not have been achieved without the participants' contribution and their enormous interest in the project. The author would like to thank all the participants who helped to collect data for eliciting strategies employed by learners of English at different proficiency levels. The project of investigating lexical strategies used by Chinese learners of English was sponsored by the grant of Chinese returning scholars from foreign countries and the grant of humanism study at Jinan University.

CHEN Long, Ph.D., College of Foreign Studies, Jinan University.
} 
the identification of strategies and the taxonomy where necessary. Since the focus of the study is on the strategies learners use to solve lexical problems rather than error analysis, the examples given in the paper

are deviant in some way, but nevertheless 'errors' should only be regarded as one kind of 'solutions'. This is another reason for calling them approximations: lexical approximations, as grammatical ones, can be correct by chance, 'covert lexical errors' in other words. (Zimmermann, 1987c, p. 58)

\section{A Taxonomy of Strategies}

The taxonomy is presented in four major categories, with subcategories for three of them. The taxonomy is not regarded as exhaustive, nor does it present the ideal way of solving lexical problems (Dörnyei \& Scott, 1997; Haastrup, 1991; Mondahl, 1995).

According to Bialystok (1983, 1984, 1990), Bongaerts and Poulisse (1989) and Poulisse (1987, 1997), the structure of the taxonomy is based on an organizing feature selected by the researcher. Thus, it must be acknowledged that the strategies could change their assigned position if another feature were selected. Hence, it may not be assumed that the proposed structure of categories is in any sense 'in the head'. A different organizing principle might conceivably restructure the groupings. It is important to emphasize that all taxonomies are descriptions of linguistic utterances but are addressed to the problem of learner behavior. On the basis of the form of language produced, the claim is that the learner has used a particular strategy. The organization of utterances in taxonomies is based on various levels of inference concerning the underlying mental processes, or behavior, that produced them. Researchers using various methodologies and studying different kinds of subjects have more or less agreed on the sorts of things that second language learners do in order to communicate (Kasper \& Kellerman, 1997; Tarone, 1980; Yule \& Tarone, 1997; Zimmermann, 1988, 1989a, 1999).

Table 1 shows an overview of the main categories of the lexical search taxonomy. What follows are definitions and examples of each category in the taxonomy. Examples provided are deviant in some way, but nevertheless 'errors' should only be regarded as one kind of ‘solutions' or lexical approximations (Zimmermann, 1987c, p. 58), since the focus of the study is on strategies learners use to solve lexical problems rather than error analysis.

Table 1

A Taxonomy of Lexical Search: Overview

\begin{tabular}{|c|c|c|c|c|}
\hline \multirow[t]{2}{*}{ Retrieval } & \multicolumn{2}{|c|}{ Strategic activities } & $\begin{array}{l}\text { Non-strategic } \\
\text { activities/Monitoring }\end{array}$ & Abandonment \\
\hline & $\begin{array}{l}\text { L1-based strategies } \\
\text { (1) Form-oriented: } \\
\text { Relexification } \\
\text { (2) Content-oriented } \\
\text { (a) L1 synonym } \\
\text { (b) L1-related concept } \\
\text { (c) L1 decomposition } \\
\text { (d) L1 paraphrase } \\
\text { (e) L1 association }\end{array}$ & $\begin{array}{l}\text { L2-based strategies } \\
\text { (1) Form-oriented: } \\
\text { L2 form } \\
\text { (2) Content-oriented } \\
\text { (a) L2 synonym } \\
\text { (b) L2-related concept } \\
\text { (c) L2 figurative expression } \\
\text { (d) Formal variation of L2 } \\
\text { concept or synonym } \\
\text { (e) L2 paraphrase } \\
\text { (f) Word coinage } \\
\text { (compounding, } \\
\text { derivation, conversion) } \\
\text { (g) L2 Association }\end{array}$ & $\begin{array}{l}\text { (1) Reflection } \\
\text { (2) Metalinguistic } \\
\text { Statements } \\
\text { (3) Deficit statement } \\
\text { (4) Orthographic check } \\
\text { (5) L1 or L2 repetition }\end{array}$ & $\begin{array}{l}\text { (1) Problem avoidance } \\
\text { (2) Strategy avoidance }\end{array}$ \\
\hline
\end{tabular}




\section{Definitions of Lexical Strategies}

Since the strategies are elicited and grouped in Table 1, it is necessary to define each strategy. When the definition of each strategy is presented, example for each strategy is also given. When the examples are presented, Chinese words which elicit lexical strategies come first and are indicated in bold letters. Literal translation of each Chinese morpheme is demonstrated in italics underneath each Chinese word. Learner's form(s) either L1 or L2 is (are) given in bold italics while the ideal/correct solutions and other English words are included in inverted commas. Then the excerpts of the think-aloud protocols or retrospection or final solutions are presented. In the excerpts, the subjects are numbered. The type of data follows the subject number. 'Retro' stands for retrospective interview, 'TAP' for think-aloud protocols and line number after colons, and ' $F$ ' for the learner's final solutions. The ideal solutions for Chinese words are put in the bracket. The researcher's rough translation can be found in the bracket beneath the whole excerpt where necessary. When analyzing the data collected in the experiment, the researcher followed certain transcription rules so that lexical strategies can be found in the think-aloud protocols and retrospection interviews. Transcription rules are shown in Table 2.

Table 2

Transcription Rules

\begin{tabular}{|l|l|}
\hline Transcribing conventions & Transcription codes \\
\hline (.X) = seconds & Pause: value \\
Underlined letters/syllabus = emphasized words or syllabuses & Emphasis: underline appearance \\
exceptionally or irregularly stressed ones & \\
Capital letters = spelling & Orthographic check \\
Repeated letters = lengthened sound & Vowel stressed: emphasis \\
$\nearrow /=$ question & Intonation: rise \\
$/ /=$ exceptional or incorrect pronunciation & Omission \\
$/ \ldots /=$ not clearly audible or omitted & Laugh/Sigh/Cough/Whisper/Throat clearance \\
Paralinguistic features & The original text is read. \\
Reading & The sound of writing \\
Writing & \\
\hline
\end{tabular}

\section{Retrieval}

This strategy indicates that the subjects have a direct path to literal equivalents in the same word class without difficulties. Example (1) shows the case.

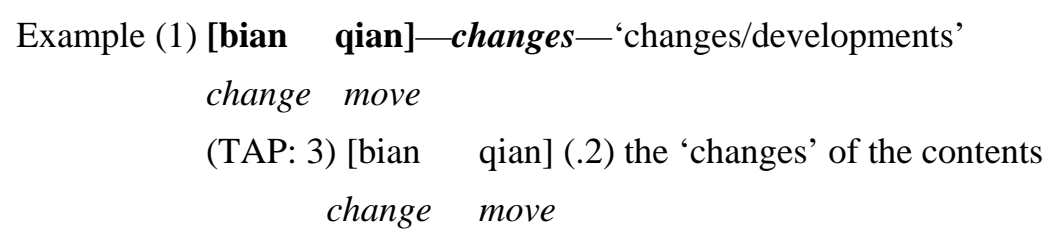

Such instances repeatedly occur in the subjects’ protocols and final solutions. Almost all subjects mentioned it. The subjects did not hesitate choosing 'changes' for [bian qian]. It is clear that the word was selected from memory out of same word class without any difficulties. Therefore, it is not considered a linguistic strategy.

\section{Strategic Activities}

Strategic behavior is distinguished from non-strategic behavior. There are two main headings in this section. L1-based strategies and L2-based strategies have form-orientation and content-orientation as subheadings. With 
respect to form-orientation and content-orientation, there seems to be neither theoretical or analytical literature nor any other scholarly references to rely on. However, "form-orientation and content-orientation occur at different levels of language, it can be L1 or L2 oriented, it is a matter of degree and there can be instances of mixed sources” (Zimmermann, 1987a, 1987b, 1987c). Before the taxonomy is explained in more detail, it is necessary to mention form-orientation and content-orientation briefly with reference to Zimmermann (1987c) and Zimmermann (1988) so as to illustrate the taxonomy more clearly, because they are two important subheadings for L1-based strategies and L2-based strategies.

When form-oriented strategies are implemented, it means that learners try to imitate the L1 or L2 form. When content-orientation strategies are used, learners usually paraphrase freely. Thus, strategies involving form-orientation and content-orientation occur at all levels, "as simplex and complex words and as phrasal renderings" (Zimmermann, 1987c, p. 61) in order to search in a semantic direction. Even though content-oriented strategies occur more often, the alternative of form-orientation is not forgotten. It is believed that form-oriented solutions cannot be obtained without considering content-orientated possibilities. Consequently, it is sometimes not possible to completely distinguish these two notions from each other (Zimmermann \& Schneider, 1987, p. 190).

L1-based strategies. L1-based strategies in the taxonomy refer to those instances when learners draw on their native Chinese in order to solve lexical problems and translate them into L2. The strategies in this category contain form-orientation and content-orientation with subcategories. In form-orientation category, there is only one strategy, relexification.

Form-orientation strategies. In this study, relexification means that the subject replaces L1 elements morpheme by morpheme with L2, maintaining the Chinese head-modifier structure, and basing his or her choice of words on other meanings of Chinese forms. Due to the language distance between Chinese and English, it is not very common for Chinese students of English to form the target word with a pure and similar structure in L2, but use more indirect form-orientation, which means that the words are searched via a loose synonym or an intermediate form erroneously taken as a synonym (Zimmermann, 1987c, p. 59) (see Example (2)).

Example (2) [xing wei] [zhun ze]—act rule—'behavioral norms'

act behave norm rule

(TAP: 34) the most people's 'act rule' $\nearrow(.2)$ [xing wei] [zhun ze]

act behave norm rule

(the most people's behavioral norms)

Content-orientation strategies. Regarding L1-based content-orientation, the following subcategories are established in the taxonomy. Examples involving L1 forms are presented in bold italics.

L1 synonym. The subjects use indirect form-orientation to get the word they want to use. Or they employ a single L1 synonym or a complex L1 synonym for this strategy. Since absolute synonym hardly exists, the synonym is used in the sense of 'near synonym'.

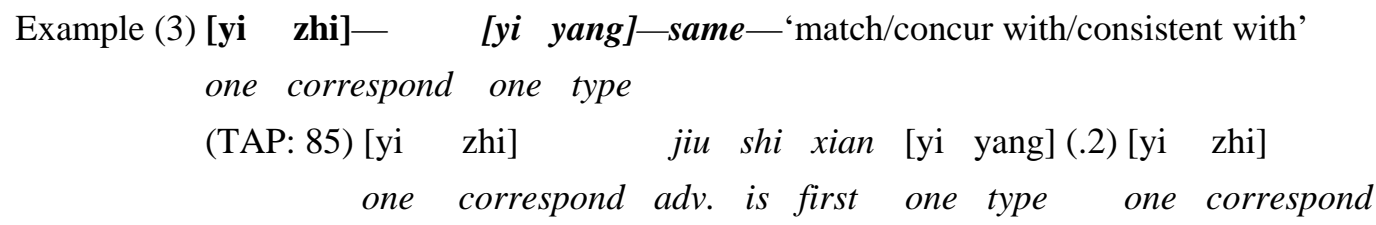




$$
\begin{aligned}
& \text { xiang dao shi same } \\
& \text { think arrive is }
\end{aligned}
$$

In Example (3), the L1 form [yi yang] is considered the synonym of [yi zhi]. The subject translated the L1 form into L2 to get 'same' in the protocol.

L1-related concept. The subjects use an L1 supernym, L1 heteronym or cohyponym.

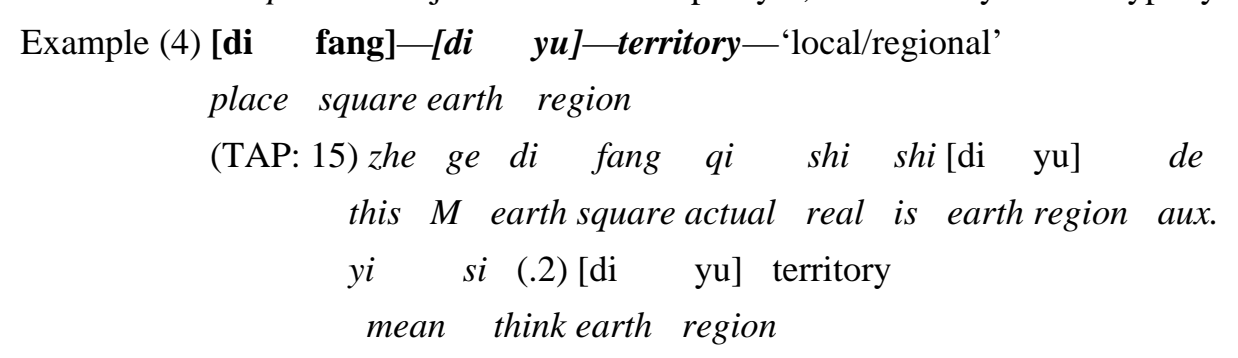

(Here it actually refers to territory)

In Example (4), 'territory' in the protocol was hit via translating the L1 form [di yu] into L2. [di yu] in Chinese is considered the supernym of [di fang].

L1 decomposition. The subjects analyze L1 lexemes morpheme by morpheme according to the related meaning.

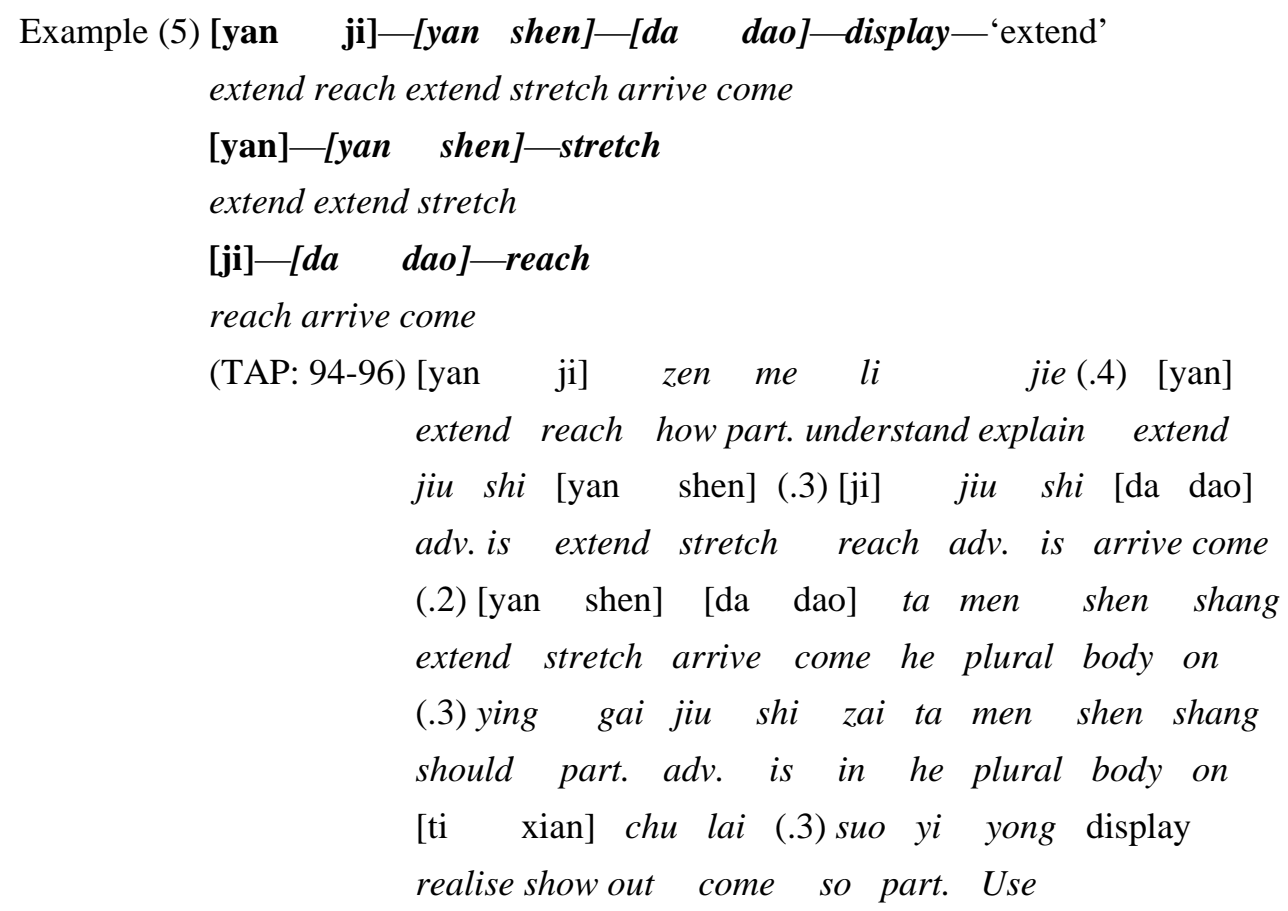

(How to understand. [yan] means 'to stretch' and [ji] means 'to reach'. 'to stretch and reach' means that something is reflected on them. Therefore, 'display' is used here.)

In Example (5), the subject analyzed the L1 form [yan ji] as [yan] which has the L1 meaning of [yan shen] and [ji] with the L1 meaning [da dao]. Thus the subject got [yan shen] [da dao] and translated into L2 form as 'display'.

L1 paraphrase. The subjects use semantic intention such as functional or formal or material characteristics. Hedged supernyms and heteronyms, or negated antonyms and heteronyms, are also employed. 


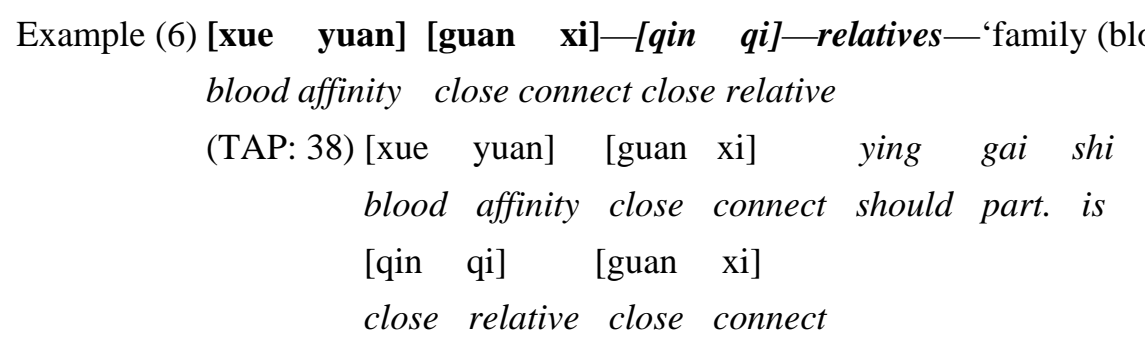

(Family ties should mean close connection of relatives)

Such instance (see Example (6)) indicates that the L1 form [qin qi] was used to explain the meaning of [xue yuan] [guan xi]. The learner's form 'relative' was obtained by paraphrasing L1.

L1 association. The subjects use personal semantic associations from L1. Even L1 word is used randomly or unclassifiably.

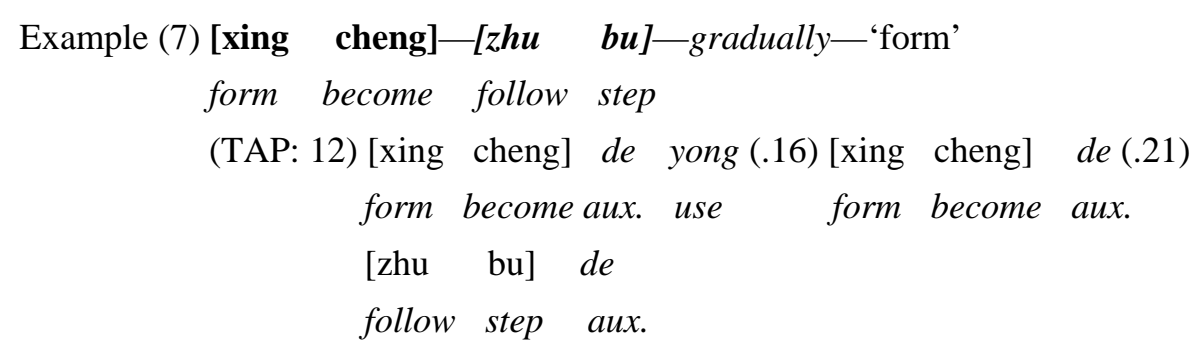

In Example (7), the subject used the L1 word [zhu bu], which is actually an adverb, to translate [xing cheng], which should be a verb in the target form. Here the L1 word was used randomly.

L2-based strategies. In addition to L1-based strategies mentioned above in the study, L2-based strategies are frequently employed by the learners of different proficiency group. L2-based strategies mean that learners rely on their L2 knowledge directly without recourse to their L1 to produce the desired lexical items. This heading also contains form-orientation and content-orientation with subcategories.

Form-orientation strategies. 'L2 form' under the heading of L2-based form-orientation in the taxonomy means that the subjects use L2-like forms, similar to existing L2 words. Example (8) illustrates the situation.

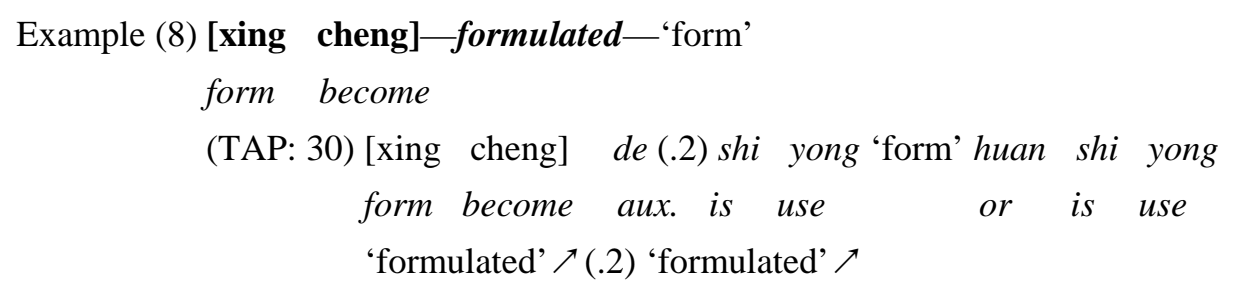

Content-orientation strategies. Similar to the use of L1-based strategies, learners of two proficiency groups also employ L2-based content-orientation strategies when they have difficulty in communication.

L2-based content-orientation strategies include L2 synonyms, L2-related concepts, L2 figurative expressions, formal variations of an L2 concept or synonym, L2 paraphrases, word formation (compounding, derivation and conversion) and L2 association in the taxonomy. Definitions of L2-based content-orientation strategies are presented below and examples are provided for each strategy.

L2 synonym. The subjects employ single L2 synonyms or complex words with a similar meaning. The use of this strategy is shown in Example (9).

Example (9) [zhun ze]—criteria-'norms' 
norm rule

(TAP: 15) [zhun ze] (.8) which is a 'criteria' (.1) for most of the people (.7) norm rule

which is a 'criteria'

L2-related concept. The subjects use L2 supernym, L2 heteronym, cohyponym or meronym. Example (10) demonstrates the case.

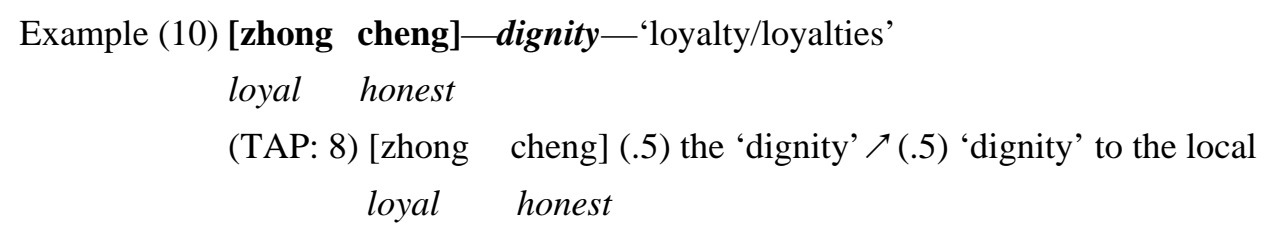

L2 figurative expression. The subjects use metaphor, metonym or simile in the target language.

Example (11) [guo jia]—motherland-'country'

country family

(TAP: 42) love 'motherland' (.2) is love (.2) people (.9) love 'motherland' is love the people the whole people

Such instance (see Example (11)) shows that the subject did not use 'country' directly in this protocol. But 'motherland' as a metaphor to indicate that 'country' was chosen.

Formal variation of an L2 concept or synonym. The subjects use existing English words which are morphologically related but do not fit into the text, since they are not from an adequate word class. Example (12) indicates the use of this strategy.

Example (12) [feng jian]-feudalism-'feudal'
confer build

(Subject 4 F) [feng jian] 'feudalism'

confer build

L2 paraphrase. The subjects use semantic intention such as functional or formal or material aspects. Hedged supernyms and heteronyms, or negated antonyms and heteronyms, are also employed (see Example (13)).

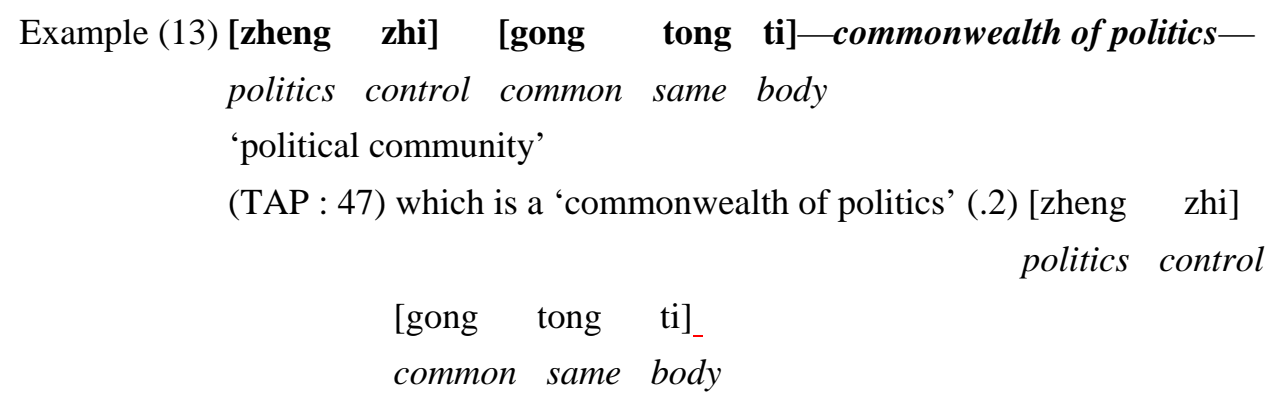

Word coinage. When learners use word coinage, they use some rules of word formation to search suitable word. In the study, compounding, derivation and conversion are used more frequently than other word formation rules.

(1) Compounding. The subjects use compounding to create non-existing words in English. Example (14) shows the use of this strategy.

Example (14) [xing wei] [zhun ze]-behavior principles—'behavioral norms' 


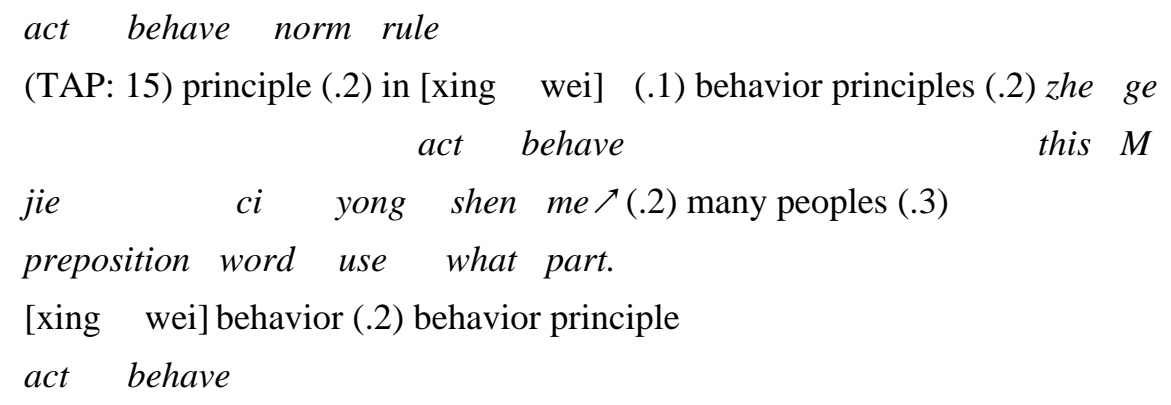

(2) Derivation. The subjects use derivation to create non-existing words in English (see Example (15)).

Example (15) [ge ju]—dividance —-'separatist regimes'

cut occupy

(TAP: 43) and why the 'dividance' of the country become very popular

(3) Conversion. The subjects use conversion to create non-existing words in English.

Example (16) [fen feng]—-titled-'confer territories and fiefs'

divide entitle

(TAP: 28) [fen feng] jiu shi (.2) 'title' (.1) 'titled' [fen feng] 'titled'

divide entitle adv is divide entitle

In Example (16), when 'title' is used as a verb, it does not have the meaning of 'confer'. In the protocol, the subject wants to indicate the meaning of 'confer' by using 'title' as a verb. Thus, it is conversion.

L2 association. The subjects use personal semantic associations of L2, resulting in a form that does not fit into the text. The words are existing English words but have no discernible semantic connection. The use of this strategy is demonstrated in Example (17).

Example (17) [deng ji]—launch—'succeeded to the throne'

climb base

(TAP: 14) [wang wei] (.13) 'launches' (.5) after he 'launched' (.3) position

king position

\section{Non-Strategic Activities/Monitoring Strategies}

Non-strategic activities, also called monitoring strategies, occur throughout the protocols across proficiency levels. Monitoring strategies in the taxonomy contain five subcategories, which are defined as follows and examples are given accordingly.

Reflection. When the subjects feel uncertain about the lexical items, they use reflection to help them find the target word. This strategy is shown in Example (18).

Example (18) (TAP: 5) zhe li neng yong deep ma

here part. can use interj.

(Can I use ‘deep' here?)

Metalinguistic statements. The subjects apply what they consider as rules according to L2 knowledge or processes of successful translation (see Example (19)).

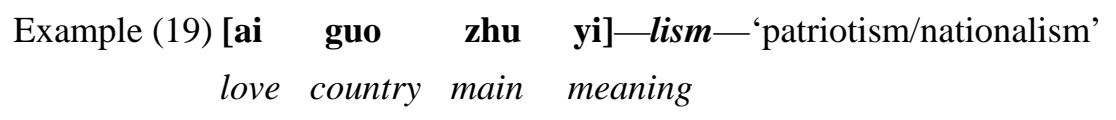




$$
\begin{aligned}
& \text { (TAP: 3) [ai guo zhu yi] (.1) ying gai shi shen me lism } \\
& \text { love country main meaning should part. is what part. } \\
& \text { ('patriotism' should be a word somewhat like '-lism') }
\end{aligned}
$$

Deficit statements. The subjects murmur the word or phrase. Almost all subjects did this when they thought aloud. Example (20) indicates the situation.

$$
\begin{aligned}
& \text { Example (20) (TAP: 6) bi jiao nan fan yi ya yong pu tong } \\
& \text { compare than difficult turn translate interj. use ordinary through } \\
& \text { dian de } \\
& \text { dot part. } \\
& \text { (well, comparatively it is difficult to translate just use a normal one) }
\end{aligned}
$$

Orthographic check. The subjects check the spelling but do not coarticulate. Then they write down the word they use.

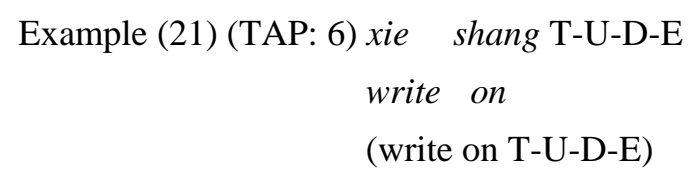

Example (22) (TAP: 53) in social S-O-C-I-A-L society social in social

Such instances (see Examples (21)-(22)) show that the subjects check their spelling of the words and then write them down when they feel the spelling is correct or when they are not very sure of the spelling.

$\mathbf{L} 1$ and $\mathbf{L} 2$ repetition. The subjects repeat the L1 and L2 words for getting the word they need. When subjects did the performance task, they kept repeating the words either in their native language or in English so as to try to access the needed words.

Example (23) (TAP: 29) $\left[\begin{array}{rrrr}f e n & \text { feng }\end{array}\right.$
divide entitle

In Example (23), when the subjects found the L1 words difficult to understand, they started repeating them until they found the words they thought appropriate. Otherwise, they abandoned the particular solutions.

However, sometimes the subjects just repeated the L2 words when they felt it was more convenient to do so in order to get lexical approximations.

Example (24) (TAP: 2) change (.3) and changes (.2) of patriotism patriotism patrioootism (.2) patriotism

In Example (24), 'change' and 'patriotism' are repeated. In fact, 'patriotism' is repeated three times. It seems to help the subjects determine the approximations.

\section{Abandonment}

There are two subcategories belonging to this type of strategy in the taxonomy. One is problem avoidance and the other is strategy avoidance.

Problem avoidance. The subjects do not want to continue, such as 'I don't know this', 'I don't want to do this.'

$$
\begin{gathered}
\text { Example (25) (TAP: 8) qiang yu (.2) bu zhi dao zen me fan yi qiang yu } \\
\text { strong than not know to how part.turn translate strong than } \\
\text { (don't know how to translate 'stronger than') }
\end{gathered}
$$




\section{Example (26) (TAP: 5) wo bu zhi dao. o, wo bu zhi dao zhe ge $I$ not knowreach interj. I not know reach this $M$ \\ (I don’t know. Well, I don’t know it.)}

Examples (25)-(26) show that the subjects gave up their efforts to find lexical approximations due to a lack of competence (and confidence).

Strategy avoidance. The subjects try to use word formation processes to find the words but give up after several trials, saying 'this is too hard for me', 'I'm not sure if it's right' (see Examples (27)-(28)).

Example (27) (TAP: 11) zhe ge tai nan wo bu xiang zuo zhe ge
this $M$ too hard $I$ not want do this $M$

(This one is too difficult. I don't want to do it)

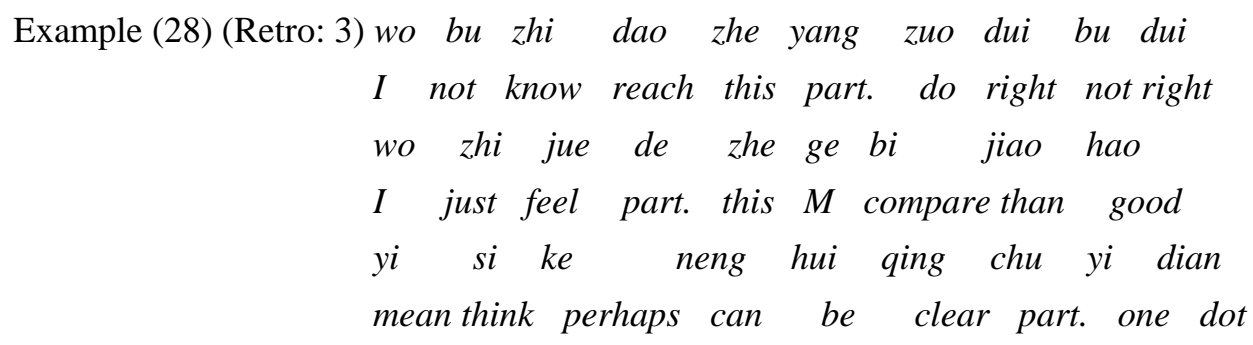

(I'm not sure if it's right. I just feel it's better here. Then the meaning could be a little clearer.)

\section{Summary}

In this paper, the taxonomy of lexical strategies employed by Chinese learners of English at different proficiency levels is identified on the basis of Zimmermann (1989b) and Poulisse, Bongaerts, and Kellerman (1990, chapter 6). The relevant taxonomy can be divided into four major categories involving linguistic and non-linguistic strategies. Linguistic strategies with subcategories containing L1-based strategies and L2-based strategies are the focus of the study. When learners know lexical strategies and use them in their communication with native speakers, they will have more confidence to communicate. When teachers of English have some ideas of these strategies employed by the learners, they can use some effective ones to help their students accordingly. Although the taxonomy can be considered the first one in the study of lexical strategies by Chinese learners of English, there are still some limitations and need to have further investigation.

\section{References}

Bialystok, E. (1983). Some factors in the selection and implementation of communication strategies. In C. Færch \& G. Kasper (Eds.), Strategies in interlanguage communication. London: Longman.

Bialystok, E. (1984). Strategies in interlanguage learning and performance. In A. Davis, C. Criper, \& A. Howatt (Eds.), Interlanguage: Papers in honor of S. Pit Corder. Edinburgh: Edinburgh University Press.

Bialystok, E. (1990). Communication strategies: A psychological analysis of second-language use. Oxford: Blackwell.

Bongaerts, T., \& Poulisse, N. (1989). Communication strategies in L1 and L2: Same or different?. Applied Linguistics, 19(3), $253-268$.

Dörnyei, Z., \& Scott, M. L. (1997). Review article: Communication strategies in a second language: Definitions and taxonomies. Language Learning, 47(1), 173-210.

Haastrup, K. (1991). Lexical inferencing procedures or talking about words. Tuebingen: Gunter Narr Verlag.

Kasper, G., \& Kellerman, E. (1997). Communication strategies: Psycholinguistic and sociolinguistic perspectives. London/New York: Longman. 
Mondahl, M. (1995). Lexical search strategies: A study of translation processes in a brief text. Multilingua, 14(2), 183-204.

Poulisse, N. (1987). Problems and solutions in the classification of compensatory strategies. Second Language Research, 3, 141-153.

Poulisse, N. (1997). Compensatory Stratgies and the principles of clarity and economy. In G. Kasper \& E. Kellerman (Eds.), Communication strategies: Psycholinguistic and sociolinguistic perspectives. London/New York: Longman.

Poulisse, N., Bongaerts, T., \& Kellerman, E. (1990). The use of compensatory strategies by Dutch learners of English. Dordrecht: Foris Publications.

Tarone, E. (1980). Communication strategies, foreign talk and repair in interlanguage. Language Learning, 30(2), 417-431.

Yule, G., \& Tarone, E. (1997). Investigating communication strategies in L2 reference: Pros and Cons. In G. Kasper \& E. Kellarman (Eds.), Communication strategies: Psycholinguistic and sociolinguistic perspectives. London: Longman.

Zimmermann, R. (1987a). Retrospective and introspective methods in the study of L2 lexical strategies. Angliestentag 1987. Giessen: Hoffman.

Zimmermann, R. (1987b). Lexical search in an 12 as impeded communication. Folia Linguistica, 21, 407-424.

Zimmermann, R. (1987c). Form-oriented and content-oriented lexical errors in L2 learners. International Review of Applied Linguistics, 25(1), 55-67.

Zimmermann, R. (1988). Paraphrase errors and word-formation errors in advanced German learners of English. In J. Klegraf \& D. Nehls, (Eds.), Essays on the English language and applied linguistics on the occasion of Gerhard Nickel's 60th birthday. Heidelberg: Gross.

Zimmermann, R. (1989a). A partial model of lexical search in L1-L2 translation. In H. W. Dechert \& M. Raupach (Eds.), Interlingual processes. Tübingen: Gunter Narr Verlag.

Zimmermann, R. (1989b). Introspective evidence for strategies of L2 lexical search. In J. Arabski (Ed.), On foreign language learning: Papers from the 2nd international conference on second/foreign language acquisition. Wroclaw: Ossolineum.

Zimmermann, R. (1999). Lexical strategies for advanced learners: A plea in favor. In M. Wysocka \& B. Leszkiewicz (Eds.), On language theory and practice. In honour of Janusz Arabski on the occasion of his 60th birthday. Language acquisition, learning and teaching (Vol. 2). Katowice: Wydawnictwo Uniwersytetu Slaskiego.

Zimmermann, R., \& Schneider, K. D. (1987). The collective learner tested: Retrospective evidence for a model of lexical search. In C. Faerch \& G. Kasper (Eds.), Introspection in second language research. Clevedon: Multilingual Matters Ltd.. 